\title{
Observation on the Anesthesia Effect of Ultrasound-Guided Nerve Block for Elderly Patients with Lower Limb Fractures
}

\author{
Lihong Cheng ${ }^{*}$, Shanzhi Luo \\ Shuyang County Central Hospital, Suqian 333600, Jiangsu Province, China
}

\begin{abstract}
[Abstract] Objective: To explore the anesthesia effect of ultrasound-guided nerve block in elderly patients with lower limb fractures. Methods: From November 2017 to November 2020, 50 elderly patients with lower limb fractures in our hospital were divided into experimental group ( 25 cases, general anesthesia + femoral nerve and sciatic nerve block) and control group (25 cases, general body anesthesia). Compare the MAP, HR, anesthesia effect, and adverse reactions between the two groups at each time period. Results: Before induction, the difference in MAP and HR between the two groups of patients did not form, $\mathrm{p}>0.05$; the MAP and HR of the experimental group were compared with the control group at the time of skin incision, 1 hour during the operation, and removal of the laryngeal mask, $\mathrm{P}<0.05$; the time of extubation in the experimental group $(14.28 \pm 3.18) \mathrm{min}$, awake time $(5.57 \pm 1.32) \mathrm{min}$, orientation recovery time (11.89 \pm 2.23$) \mathrm{min}$, propofol dosage (191.36 \pm 22.48$) \mathrm{mg}$, remifentanil dosage $(0.23 \pm 0.04) \mathrm{mg}$, Compared with the control group, $\mathrm{P}<0.05$; the adverse reaction rate of the experimental group $(8 \%, 2 / 25)$ was lower than that of the control group $(32 \%, 8 / 25), \mathrm{P}<0.05$. Conclusion: The use of ultrasound-guided femoral nerve and sciatic nerve block for elderly patients with lower limb fractures can enhance the effect of anesthesia, effectively reduce the use of anesthetics, and have fewer adverse reactions. It is worthy of promotion.
\end{abstract}

Key words: Ultrasound guidance; Nerve block; Lower limb fracture; Anesthesia effect

Publication date: May, 2021; Publication online: 31 May, 2021

*Corresponding author: Lihong Cheng, 1723828668@qq.com

The effect of combined spinal-epidural anesthesia for patients with lower limb fractures is ideal and will not affect the cognitive function of the patients. However, there are still some elderly patients who have increased the difficulty of puncture due to the calcification of the ligamentum flavum and bone hyperplasia, and the requirements for intraspinal anesthesia are sideways. Lying position. However, patients with fractures cannot tolerate it because of the strong pain, so it is not suitable for all patients ${ }^{[1]}$. In addition, general anesthesia with endotracheal intubation can affect the metabolism of drugs and prolong the recovery time after surgery. The use of ultrasound-guided femoral nerve and sciatic nerve block for patients can significantly enhance the anesthesia effect, so it is widely used in anesthesia programs for elderly patients with lower limb fractures. It can be seen that in-depth study and analysis of the anesthesia effect of nerve tissue in elderly patients with lower limb fractures under the guidance of ultrasound has certain practical significance.

\section{Materials and methods}

\subsection{Basic information}

The subject selected 50 patients with lower limb fractures who were treated in our hospital from November 2017 to November 2020 for statistical comparison. Two groups were divided into two groups according to the admission serial number. There were 25 cases in the control group, 16 males and 9 females, age range between 54 and 82 years old, the median age is $(66.24 \pm 1.56)$ years old, the experimental group has 25 cases, 18 males and 7 females, the age range is 
between 51 to 80 years old, and the median age is $(66.26 \pm 1.51)$ years old. The data of the two groups of patients showed $\mathrm{p}>0.05$, and the comparability was significant.

\subsection{Methods}

The control group was given general anesthesia. After entering the operating room, the patients were monitored for their vital signs. Changtonin, midazolam, propofol, fentanyl, and cis-atracurium were slowly injected intravenously In patients, the specific doses are $0.5 \mathrm{mg}, 0.05 \mathrm{mg} / \mathrm{kg}, 1-1.5$ $\mathrm{mg} / \mathrm{kg}, \quad 3 \mu \mathrm{g} / \mathrm{kg}, \quad 0.15 \mathrm{mg} / \mathrm{kg}$ to achieve anesthesia induction $^{[2]}$. Use the mask to give oxygen, and when the consciousness disappears and the muscle relaxation is satisfied, put a moderately sized laryngeal mask into it, and connect it to the anesthesia machine to control breathing.

The experimental group was treated with general anesthesia + femoral nerve and sciatic nerve block. After general anesthesia, the bone nerve and sciatic nerve block were deployed under ultrasound guidance. Among them, the femoral nerve block required the patient to be in a supine position. After routine disinfection and draping, the puncture point was $2 \mathrm{~cm}$ below the line of the pubic tubercle and the anterior superior iliac spine and $2 \mathrm{~cm}$ outside the femoral artery pulse. In the case of blood-free withdrawal, $15 \mathrm{ml}$ of ropivacaine hydrochloride with a concentration of $0.375 \%$ (National Medicine Standard: H20203032 Approval Date: 2020-02-20 Manufacturer: Shan Dongfang Ming Pharmaceutical Group Co., Ltd. English name: Ropivacaine Hydrochloride Injection) slowly injected $\mathrm{it}^{[3]}$. In sciatic nerve block, the patient's affected side should be in a lateral position. After routine disinfection and draping, the position of the puncture under direct ultrasound vision was observed, and the concentration of $15 \mathrm{ml}$ was set under the condition of no blood. $0.375 \%$ ropivacaine hydrochloride was slowly injected into it.
During the surgical treatment, both groups of patients were injected with propofol (National Medicine Standard: H20093542 Approval Date: 2013-12-06 Manufacturer: Hebei Yipin Pharmaceutical Co., Ltd. English Name: Propofol) and Remifentanil (National Medicine Standard) Word: H20143315 Date of Approval: 2014-09-30 Manufacturer: Jiangsu Enhua Pharmaceutical Co., Ltd. English name: Remifentanil Hydrochloride for Injection), the dosage standards were $1-8 \mathrm{mg} /(\mathrm{kg} \cdot$ hour $), \quad 0.05$ $\mu \mathrm{g} /(\mathrm{Kg} \cdot \mathrm{hour})$, at the same time intermittent intravenous injection of cis-atracurium $5 \mathrm{mg}$, to ensure that the bispectral index of EEG is between 40-60 $0^{[4]}$. You can stop pumping drugs 5 minutes before the end of the operation, and pull out the laryngeal mask when the patient had a clear consciousness and resumes spontaneous breathing. If the patient's blood pressure drops or increases by more than $20 \%$ of the basic value during the operation, rehydration and symptomatic treatment are required ${ }^{[5]}$. If the patient's heart rate is less than 50 beats per minute, atropine should be used to ensure the safety of their lives.

\subsection{Evaluation index}

1.3.1 The MAP and HR of the patients in each time period was evaluated.

1.3.2 The anesthesia effects and adverse reactions between the two groups were compared.

\subsection{Statistical analysis}

The statistical software SPSS19.0 processes the data of the two groups, and $\mathrm{P}<0.05$ means that the data has clinical statistical significance.

\section{Results}

\subsection{Analyse of the MAP and HR in each period of the experimental group and the control group}

After induction, MAP and HR were compared in each period between groups, $\mathrm{P}<0.05$ (Table 1).

Table 1. Comparison of MAP and HR in each period of the two groups ( $\overline{\mathrm{X}} \pm \mathrm{s}$ )

\begin{tabular}{|c|c|c|c|c|c|c|c|c|c|}
\hline \multirow[b]{2}{*}{ Group } & \multirow[b]{2}{*}{$\mathbf{n}$} & \multicolumn{4}{|c|}{ MAP } & \multicolumn{4}{|c|}{ HR } \\
\hline & & $\begin{array}{c}\text { Before } \\
\text { induction }\end{array}$ & $\begin{array}{c}\text { Cutting the } \\
\text { skin }\end{array}$ & $\begin{array}{c}\text { Intraoperative } \\
1 \mathrm{~h}\end{array}$ & $\begin{array}{c}\text { Remove the } \\
\text { laryngeal } \\
\text { mask } \\
\end{array}$ & $\begin{array}{c}\text { Before } \\
\text { induction }\end{array}$ & $\begin{array}{l}\text { Cutting } \\
\text { the skin }\end{array}$ & $\begin{array}{c}\text { Intraoperative } \\
1 \mathrm{~h}\end{array}$ & $\begin{array}{c}\text { Remove the } \\
\text { laryngeal } \\
\text { mask } \\
\end{array}$ \\
\hline $\begin{array}{l}\text { Test } \\
\text { group }\end{array}$ & 25 & $95.47 \pm 12.34$ & $97.13 \pm 13.22$ & $96.11 \pm 12.09$ & $99.43 \pm 13.37$ & $55.47 \pm 8.83$ & $56.46 \pm 9.32$ & $59.11 \pm 9.54$ & $56.64 \pm 8.21$ \\
\hline $\begin{array}{l}\text { Control } \\
\text { group }\end{array}$ & 25 & $95.45 \pm 12.31$ & $\begin{array}{c}110.04 \pm 12.3 \\
4\end{array}$ & $109.98 \pm 13.25$ & $110.17 \pm 13.76$ & $57.27 \pm 9.24$ & $67.05 \pm 8.65$ & $69.99 \pm 8.04$ & $73.01 \pm 7.85$ \\
\hline
\end{tabular}




\begin{tabular}{llllllllll} 
T value & 0.0057 & 3.5694 & 3.8663 & 2.7989 & 0.7042 & 4.1642 & 4.3603 & 7.2057 \\
P value & 0.9954 & 0.0008 & 0.0003 & 0.0074 & 0.4847 & 0.0001 & 0.0001 & 0.0000 \\
\hline
\end{tabular}

2.2 Study on the anesthesia effects of the two groups of patients
Compared with the control group, each index of the experimental group was $\mathrm{P}<0.05$ (Table 2).

Table 2. Comparison of anesthesia effects between the experimental group and the control group ( $\overline{\mathrm{X}} \pm \mathrm{S}$ )

\begin{tabular}{ccccccc}
\hline Group & $\mathbf{n}$ & Extubation time & $\begin{array}{c}\text { Awake } \\
\text { time }\end{array}$ & $\begin{array}{c}\text { Orientation } \\
\text { recovery time }\end{array}$ & Propofol dosage & $\begin{array}{c}\text { Remifentanil } \\
\text { dosage }\end{array}$ \\
\hline Test group & 25 & $14.28 \pm 3.18$ & $5.57 \pm 1.32$ & $11.89 \pm 2.23$ & $191.36 \pm 22.48$ & $0.23 \pm 0.04$ \\
& & $18.34 \pm 4.07$ & $10.32 \pm 2.1$ & & & \\
Control group & 25 & 8 & $31.67 \pm 2.77$ & $337.28 \pm 50.98$ & $0.48 \pm 0.01$ \\
T value & & 3.9303 & 9.3192 & 27.8114 & 13.0949 & 30.3170 \\
P value & & 0.0003 & 0.0000 & 0.0000 & 0.0000 & 0.0000 \\
\hline
\end{tabular}

2.3 Comparison of adverse reactions between the Index comparison between groups, $\mathrm{P}<0.05$ (Table 3 ). experimental group and the control group

Table 3. Analysis of adverse reactions in the two groups (n/\%)

\begin{tabular}{cccccc}
\hline Group & n & Increased heart rate & Hypertension & Low blood pressure & Total incidence \\
\hline Test group & 25 & 0 & 0 & 2 & 8 \\
Control group & 25 & 3 & 4 & 1 & 32 \\
X2 & & & & & 4.5000 \\
P & & & & 0.0338 \\
\hline
\end{tabular}

\section{Discussion}

The elderly are the main group of lower limb fractures. Because of their reduced cardiovascular compensatory function, they are prone to obvious hemodynamic fluctuations during surgery and anesthesia ${ }^{[6]}$. However, conducting nerve tissue under the guidance of B-ultrasound can significantly enhance the effect of lower limb fracture surgery. Compared with blind detection operation or nerve block guided by nerve stimulator, the advantages of B-ultrasound guidance are very prominent, which are concentrated in the following aspects.

First, the positioning is more precise. Under the condition of direct vision, the direction and position of the puncture needle can be observed to ensure the accuracy of the needle, and the needle can be smoothly inserted to the nerve to be blocked;

Second, the scope of injection can be observed and adjusted to better strengthen the anesthesia effect;

Third, it is safer. Nerve tissue guided by B-ultrasound can effectively avoid damage to blood vessels and nerves ${ }^{[7]}$.
In lower limb fracture surgery, the femoral nerve and sciatic nerve block are guided by B-ultrasound, and the operation site will not be completely blocked. Therefore, it is required to be combined with general anesthesia with a laryngeal mask to enhance the anesthesia effect.

In the study, patients in the experimental group were treated with general anesthesia + femoral nerve and sciatic nerve block. Compared with the control group, $\mathrm{P}<0.05$. This proves that the combination of general anesthesia and femoral nerve and sciatic nerve block can significantly reduce the amount of anesthetic drugs used, shorten the time for patients to wake up, and have more stable hemodynamics.

Due to the intense pain in the fracture area, combined with the stimulation of surgery and anesthesia, patients with lower limb fractures make their body's stress response too strong, which activates the internal and external coagulation systems and presents a hypercoagulable state. According to relevant research results, it is found that the daily activities of fracture patients are reduced, and the vascular endothelium is damaged, and their blood flow rate is also 
slowed down. Once in a hypercoagulable state, it is easy to induce thrombosis in the lower limbs. After the thrombus falls off, a series of adverse events will be induced ${ }^{[8]}$. In clinical practice, the combined use of laryngeal mask general anesthesia and nerve block is far better than simple general anesthesia, and it will not irritate the patient too significantly, and it is not easy to cause severe blood hypercoagulability.

In general, ultrasound-guided femoral nerve and sciatic nerve block can significantly improve the anesthesia effect of elderly patients undergoing lower limb fracture surgery, and can ensure the stability of the patient's hemodynamics during the operation, and reduce the use of drugs as much as possible, which is beneficial to patients Wake up as soon as possible and reduce the incidence of adverse reactions, so it has high clinical promotion and application value.

\section{References}

[1] Wang J. Observe the anesthesia effect of ultrasound-guided femoral nerve and sciatic nerve block for elderly patients with lower limb fractures and its influence on blood coagulation status $[\mathrm{J}]$. Health Care Guide, 2021(4):264.

[2] Wang W. The effect of ultrasound-guided femoral nerve and sciatic nerve block on the anesthesia effect of elderly patients with lower limb fractures and the analysis of its influence on blood coagulation status [J]. Chinese Traumatology and Disability Medicine, 2021, 29(4): 49- 51.
[3] Su LH, He CY, Bai W, et al. To explore the effect of ultrasound-guided femoral nerve and sciatic nerve block on the anesthesia effect and blood coagulation status of elderly patients with lower limb fractures [J]. Frontiers of Medicine, 2020, 10( 7): 126-127.

[4] Lin SL. The clinical effect of ultrasound-guided lower lumbar plexus combined with sciatic nerve block anesthesia in elderly lower limb orthopedic surgery $[\mathrm{J}]$ Imaging Research and Medical Application, 2020, 4(16): 216-218.

[5] Ning XY, Zhang HY, Guo W, et al. Observation of the anesthesia effect of ultrasound-guided nerve block for elderly patients with lower limb fractures [J]. Medical Journal of National Defending Forces in Southwest China, 2019, 29(2): 119-121.

[6] Yang AL. The effect of ultrasound-guided nerve block anesthesia on the circulatory system and recovery time of elderly patients with lower limb fractures $[\mathrm{J}]$. Qingdao Medical Health, 2020, 52(1): 9-11.

[7] Zhang NN, Gao TM. The effect of general anesthesia with laryngeal mask combined with ultrasound-guided nerve block in elderly patients with lower limb fracture surgery [J]. Health Must Read, 2020(33):109.

[8] Zhang XM. Analysis of the application effect of laryngeal mask with spontaneous breathing and general anesthesia combined with ultrasound-guided nerve block in elderly patients with lower limb fracture surgery $[\mathrm{J}]$. Electronic Journal of Integrated Traditional Chinese and Western Medicine Cardiovascular Diseases, 2019, 7(33): 63. 